\title{
PET/CT-positive brown tumour - a potentially misleading finding in the evaluation of a patient for malignant primary tumour or metastases
}

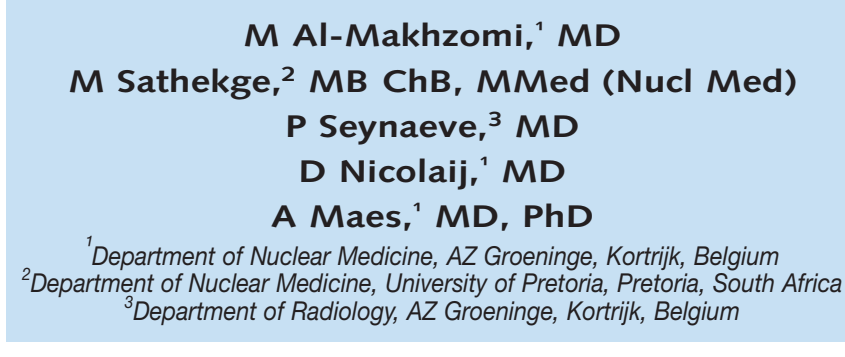

Abstract

Brown tumours are the end stage of primary or secondary hyperparathyroidism. Clinically, brown tumours most often manifest as slowly growing, painful masses. These tumours can behave aggressively and be destructive. We report a patient with high accumulation of fluorodeoxyglucose (FDG) positron emission tomography/computed tomography (PET/CT) in brown tumours as the potential cause of false-positive results in the evaluation of a patient for malignant primary tumour or metastases.

\section{Case report}

A 49-year-old man came to the hospital with a long-term history of painful right lower leg after trauma. The initial examination was unremarkable.

X-ray of the right tibia demonstrated a cystic lesion in the middle third.

Magnetic resonance imaging (MRI) demonstrated a T1-hypointense mass lesion with significant enhancement in the subcortical medulla with scalloping of the internal cortical rim. The T2-weighted images demonstrated a hypointense aspect of the centre of the lesion with some hyperintensity around the lesion. In the differential diagnosis chondromyxoid fibroma and osteitis fibrosa cystica were considered (Figs 1 a \& b).

Tc-99m methylene diphosphate (MDP) bone scintigraphy revealed abnormal uptake in the right tibial diaphysis, the proximal femur bilaterally, the sacrum and the right acetabulum.

The multifocality on bone scan raised the suspicion of malignancy.

The patient was referred for biopsy of the lesion in the right leg and a whole body positron emission tomography/computed tomography (PETCT) scan with 18F-FDG was performed. The CT scan revealed multifocal osteolytic lesions in both femora, the right acetabulum, both iliac bones, the right acromioclavicular joint and the left pubic bone (Fig. 2).A nodular structure inferior to the left lobe of the thyroid gland was discovered. The PET scan showed increased FDG uptake in the osteolytic lesions (Fig. 2). There was no increased FDG-uptake in the nodular structure inferior to the left lobe of the thyroid gland. The PET-CT results were in concordance with the pre-assumption of metastatic bone lesions. The biopsy results however revealed no signs of malignancy.
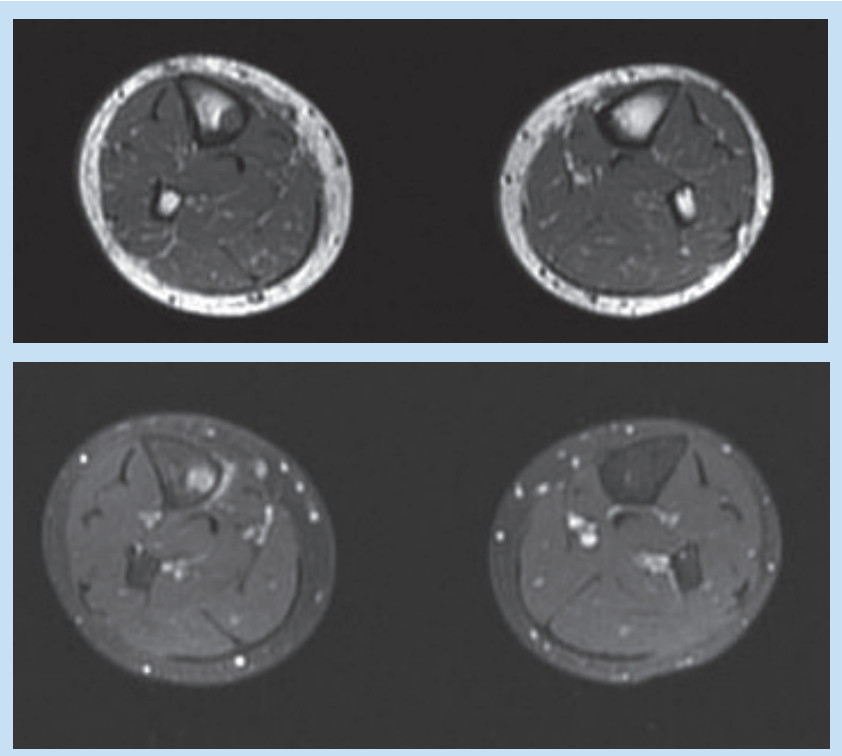

Fig. 1. Axial T1 (a) and axial postcontrast T1-weighted (b) MR image with fat suppression demonstrates a hypointense mass lesion at the corticomedullary junction of the tibial diaphysis. There is scalloping of the internal cortical rim without penetration of the cortex. Significant contrast enhancement in the mass lesion and some reactive enhancement in the adjacent periosteum and soft tissues is noted.

In view of the lesion inferior to the left lobe of the thyroid, the patient was subsequently evaluated for hyperparathyroidism.

His serum calcium level was $3.21 \mathrm{mmol} / \mathrm{l}$ (normal range: $2.10-2.55$ $\mathrm{mmol} / \mathrm{l}$ ). His alkaline phosphatase concentration was $241 \mathrm{IU} / \mathrm{l}$ (normal range: 40 - $150 \mathrm{IU} / \mathrm{l}$ ), and his parathyroid hormone level was $682 \mathrm{pg} / \mathrm{ml}$ (normal range: $12-72 \mathrm{pg} / \mathrm{ml}$ ).

Tc-99m methoxy isobutyl isonitrile (MIBI) parathyroid scintigraphy revealed a parathyroid adenoma inferior to the left thyroid lobe (Fig. 3) and ultrasonography showed a $6 \times 9 \mathrm{~mm}$ nodule of the inferior left parathyroid gland. Parathyroidectomy was undertaken and histopathological examination revealed the presence of a parathyroid adenoma. Postoperatively, plasma parathyroid hormone and calcium levels normalised. Based on these results, the osteolytic FDG avid bone lesions were classified as multiple brown tumours caused by primary hyperparathyroidism.

\section{Discussion}

This case demonstrates that increased FDG uptake in clinically occult brown tumours can be misleading in the evaluation of a patient for malignancy. Kuwahara et al. ${ }^{1}$ have recently demonstrated a case of increased FDG uptake in brown tumours with primary hyperparathyroidism.

Brown tumours of bone or osteoclastomata are highly vascular, lytic bone lesions representing a reparative cellular process rather than a neoplastic process usually seen in patients with hyperparathyroidism with hypercalcaemia. ${ }^{2,3}$ These tumours occur less frequently than in the past, because of earlier detection of hypercalcaemia., 


\section{CASE REPORT}

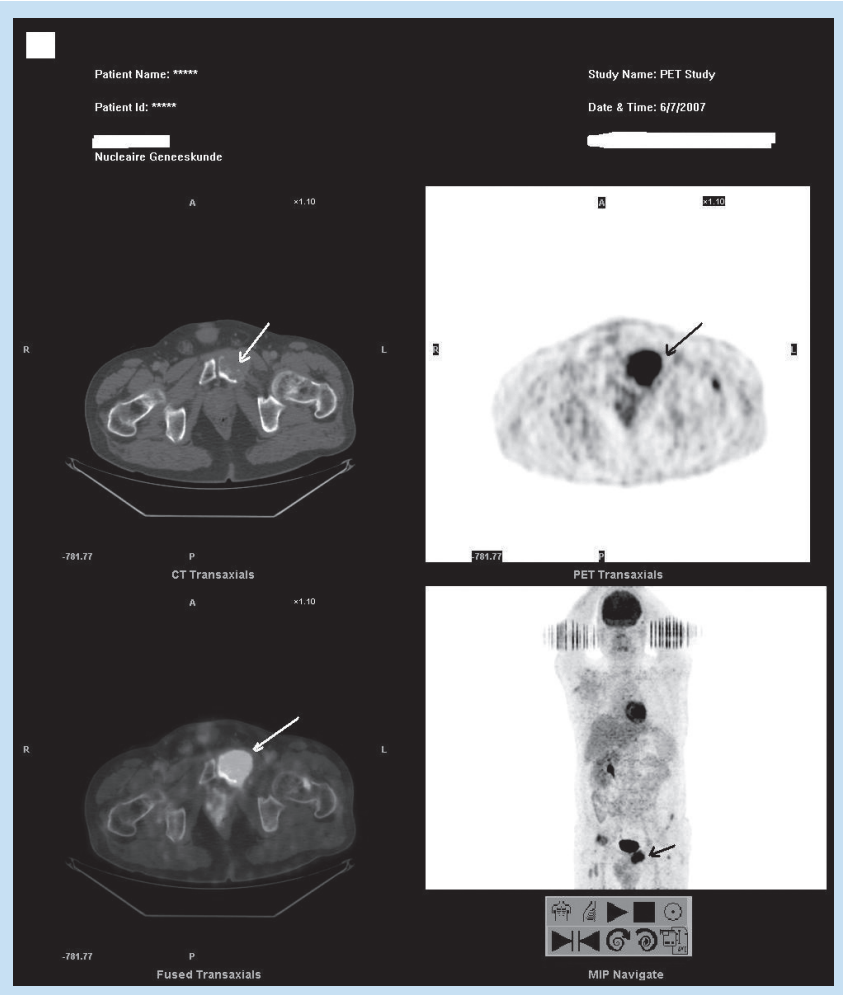

Fig. 2. CT scan of the pelvis shows a large lytic mass associated with softtissue swelling in the left pubic bone (upper left photo: white arrow). PET scan: shows high uptake in the left pubic bone lesion (upper right photo and lower right photo: black arrow). Fusion image: shows the exact site of high FDG-uptake (lower left photo: white arrow).

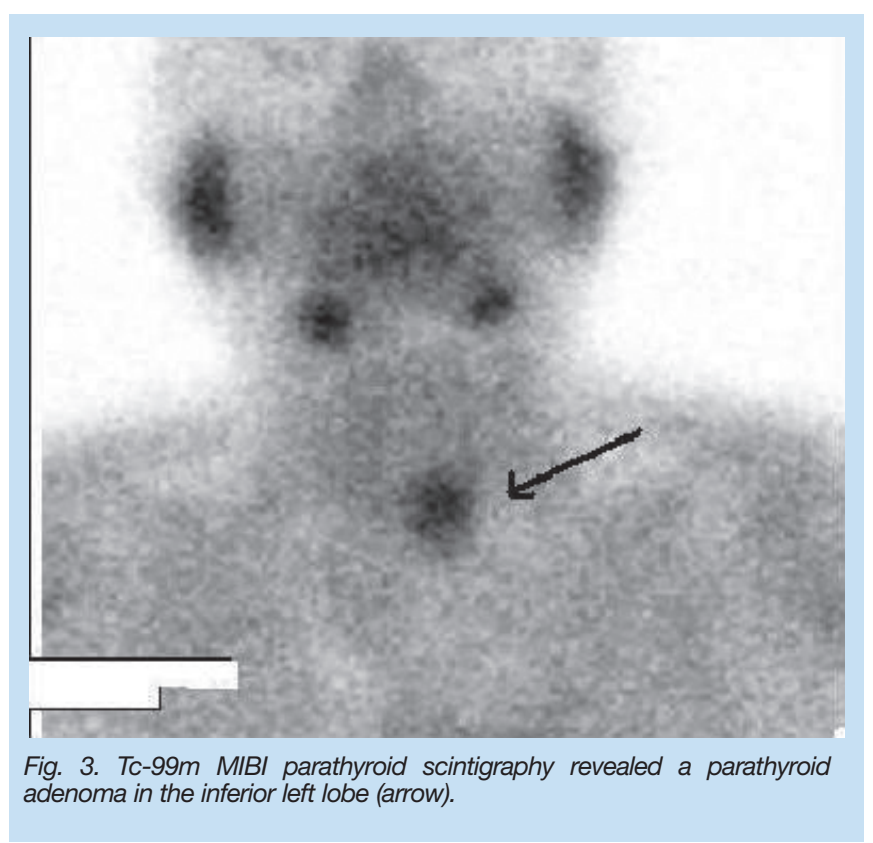

The lesion localises in areas of intense bone resorption, and the bone defect becomes filled with fibroblastic tissue that can deform the bone and simulate a neoplastic process. ${ }^{5}$ These tumours have a brown or yellow hue. ${ }^{6}$

Skeletal changes may represent the first manifestations of hyperparathyroidism, with a loss of cortical bone and an increase in trabecular bone. Bone pain and arthralgia are the most common symptoms, such as in our case.

Brown tumours exhibit no pathognomonic histological changes. Findings may include a dense fibroblastic stroma, areas of cystic degeneration, osteoid, microfractures, haemorrhage, macrophages with hemosiderin, and multinucleated osteoclastic giant cells. Similar changes may occur in fibrous dysplasia, true giant-cell tumours, and reparative granulomas. ${ }^{5}$ Histologically, brown tumours may be indistinguishable from giant-cell tumours of the bone, and correlation with clinical findings and imaging studies is essential in making the correct diagnosis.

The presence of giant cells has been suggested as partially contributing to the high uptake of FDG in benign bone lesions such as brown tumours. $^{7-9}$ Another supporting mechanism for the elevated FDG uptake is that macrophages play a central role in the host response to injury, and their energy is predominantly supplied by means of intracellular glucose metabolism. $^{10,11}$

In conclusion, PET-CT readers should be aware of the high accumulation of FDG in brown tumours as a potential cause of false-positive results in the evaluation of a patient for malignant primary tumour or metastases.

1. Kuwahara K, Izawa S, Murabe $\mathrm{H}$, et al. Increased 18F-fluorodeoxyglucose uptake in a brown tumor in a patient with primary hyperparathyroidism. J Clin Endocrinol Metab 2007; 92:2408-2409.

2. Chew F S, Huang-Hellinger F. Brown tumor. AJR 1993; 160:752.

3. Yang C, Seabold JE, Gurll NJ. Brown tumor of bone: a potential source of false-positive Thallium-201 localization. J Nucl Med 1989; 30: 1264-1267

4. Nassar GM, Ayus JC. Images in clinical medicine. Brown tumor in end stage renal disease. N Engl J Med 1999; 341: 1652.

5. Keyser IS, Postma GN. Brown tumor of the mandible. Am J Otolaryngol 1996; 17: 407-410.

6. Friedman WH, Pervez N, Schwartz AE. Brown tumor of the maxilla in secondary hyperparathyroidism. Arch Otolaryngol 1974; 100: 157-159.

7. Johnston J. Giant cell tumor of bone: the role of the giant cell in orthopedic pathology. Orthop Clin North Am 1977; 8: 751-770.

8. Ling L, Klein MJ, Sissons HA, Steiner GC, Winchester RJ. Expression of Ia and monocyte-macrophage lineage antigens in giant cell tumor of bone and related lesions. Arch Pathol Lab Med 1988; 112: 65-69.

9. Aoki J, Watanaba H, Shinozaki T, et al. FDG PET of primary benign and malignant bone tumors: standardized uptake value in 52 lesions. Radiology $2001 ; 219: 774-777$.

10. Meszaros K, Lang CH, Bagby GJ, Spitzer JJ. Contribution of different organs to increased glucose consumption after endotoxin administration. J Biol Chem 1987; 262: 10965-10970.

11. Gamelli RL, Liu H, He LK, Hofmann CA. Augmentations of glucose uptake and glucose transporter-1 in macrophages following thermal injury and sepsis in mice. J Leukoc Biol 1996; 59: 639-647. 